University of Wollongong

Research Online

Faculty of Engineering and Information

Faculty of Engineering and Information

Sciences - Papers: Part A

Sciences

$1-1-2013$

On the evolution and modelling of lattice strains during the cyclic loading of TWIP steel

\author{
Ahmed A. Saleh \\ University of Wollongong, asaleh@uow.edu.au \\ Elena V. Pereloma \\ University of Wollongong, elenap@uow.edu.au \\ Bjorn Clausen \\ Los Alamos National Laboratory \\ Donald W. Brown \\ Los Alamos National Laboratory \\ Carlos N. Tome \\ Los Alamos National Laboratory
}

See next page for additional authors

Follow this and additional works at: https://ro.uow.edu.au/eispapers

Part of the Engineering Commons, and the Science and Technology Studies Commons

Research Online is the open access institutional repository for the University of Wollongong. For further information contact the UOW Library: research-pubs@uow.edu.au 


\title{
On the evolution and modelling of lattice strains during the cyclic loading of TWIP steel
}

\begin{abstract}
The evolution of lattice strains in fully annealed $\mathrm{Fe}-24 \mathrm{Mn}-3 \mathrm{Al}-2 \mathrm{Si}-1 \mathrm{Ni}-0.06 \mathrm{C}$ twinning-induced plasticity (TWIP) steel is investigated via in situ neutron diffraction during cyclic (tension-compression) loading between strain limits of $\pm 1 \%$. The pronounced Bauschinger effect observed upon load reversal is accounted for by a combination of the intergranular residual stresses and the intragranular sources of back stress, such as dislocation pile-ups at the intersection of stacking faults. The recently modified elasto-plastic self-consistent (EPSC) model which empirically accounts for both intergranular and intragranular back stresses has been successfully used to simulate the macroscopic stress-strain response and the evolution of the lattice strains. The EPSC model captures the experimentally observed tension-compression asymmetry as it accounts for the directionality of twinning as well as Schmid factor considerations. For the strain limits used in this study, the EPSC model also predicts that the lower flow stress on reverse shear loading reported in earlier Bauschinger-type experiments on TWIP steel is a geometrical or loading path effect.
\end{abstract}

\section{Keywords}

loading, cyclic, during, strains, lattice, modelling, evolution, twip, steel

\section{Disciplines}

Engineering | Science and Technology Studies

\section{Publication Details}

Saleh, A. A., Pereloma, E. V., Clausen, B., Brown, D. W., Tome, C. N. \& Gazder, A. A. (2013). On the evolution and modelling of lattice strains during the cyclic loading of TWIP steel. Acta Materialia, 61 (14), 5247-5262.

\section{Authors}

Ahmed A. Saleh, Elena V. Pereloma, Bjorn Clausen, Donald W. Brown, Carlos N. Tome, and Azdiar A. Gazder 


\title{
ON THE EVOLUTION AND MODELLING OF LATTICE STRAINS DURING THE CYCLIC LOADING OF TWIP STEEL
}

\author{
Ahmed A. Saleh ${ }^{*}$, Elena V. Pereloma ${ }^{1,2}$, Bjørn Clausen³, Donald W. Brown ${ }^{4}$, \\ Carlos N. Tomé4, Azdiar A. Gazder ${ }^{1,2}$ \\ ${ }^{1}$ School of Mechanical, Materials and Mechatronic Engineering, University of Wollongong, \\ New South Wales 2522, Australia \\ 2Electron Microscopy Centre, University of Wollongong, New South Wales 2519, Australia \\ ${ }^{3}$ Los Alamos Neutron Science Center, Los Alamos National Laboratory, \\ New Mexico 87545, United States \\ ${ }^{4}$ Materials Science and Technology Division, Los Alamos National Laboratory, \\ New Mexico 87545, United States
}

\begin{abstract}
The evolution of lattice strains in fully annealed Fe-24Mn-3Al-2Si-1Ni-0.06C TWinning Induced Plasticity (TWIP) steel is investigated via in-situ neutron diffraction during cyclic (tensioncompression) loading between strain limits of $\pm 1 \%$. The pronounced Bauschinger effect observed upon load reversal is accounted for by a combination of the intergranular residual stresses and the intragranular sources of back stress such as dislocation pile-ups at the intersection of stacking faults. The recently modified Elasto-Plastic Self-Consistent (EPSC) model which empirically accounts for both intergranular and intragranular back stresses has been successfully used to simulate the macroscopic stress-strain response and the evolution of the lattice strains. The EPSC model captures the experimentally observed tension-compression asymmetry as it accounts for the directionality of twinning as well as Schmid factor considerations. For the strain limits used in this study, the EPSC model also predicts that the lower flow stress on reverse shear loading reported in earlier Bauschinger-type experiments on TWIP steel is a geometrical or loading path effect.
\end{abstract}

Keywords: TWIP steel, Neutron diffraction, lattice strain, Bauschinger effect, EPSC.

\section{* Corresponding author:}

\section{Ahmed A. Saleh}

School of Mechanical, Materials and Mechatronic Engineering

University of Wollongong, New South Wales 2522, Australia

Phone: +61 42213034 | Fax: +61 42213662 | e-mail: asaleh@uow.edu.au 


\section{Introduction}

TWinning Induced Plasticity (TWIP) steels containing 25-35 wt.\% Mn with small additions of Al and Si have been developed as a promising material for automotive applications due to their characteristically extended period of work hardening under applied macroscopic (or type- $I$ ) stress [1]. TWIP steels comprise a stable face centred cubic (fcc) austenite phase with low stacking fault energy between $18-40 \mathrm{~mJ} / \mathrm{m}^{2}$. This low stacking fault energy initiates twinning along with dislocation glide during room temperature deformation.

Twinning affects the high work hardening rate through either of two predominant mechanisms: (i) isotropic hardening or the "Dynamic Hall-Petch effect" or, (ii) kinematic hardening or the "Bauschinger effect". In addition to lattice friction and forest hardening effects, isotropic hardening in TWIP steels has been ascribed to reductions in the mean free path of dislocations caused by the twin boundaries acting as obstacles to further glide [2-4]. On the other hand, kinematic hardening has been attributed to the presence of an internal forward stress on the twins and an internal back stress on the matrix [2, 4]. In this regard, while several experimental and theoretical investigations have correlated the strain hardening behaviour of low stacking fault energy materials with their twinning activity in terms of the isotropic hardening effect $[3,5,6]$, limited details are available on the contribution of kinematic hardening or the Bauschinger effect to the overall strain hardening $[2,4]$.

The Bauschinger effect is generally manifested by a lowering of the yield stress upon load reversal and an extended elasto-plastic transition region. The phenomenon is generally explained through internal stress and/or dislocation -based theories [7]. The Bauschinger effect was initially ascribed to internal stresses and macroscopic residual stresses arising from the inhomogeneous deformation of individual grains [8] due to the anisotropy in their elastic moduli and yield strength with orientation and/or different phases [9]. Such internal stresses that self-equilibrate at length scales comparable to the grain size are known as intergranular or type-II stresses [10].

Thereafter, dislocation-based theories introduced by Mott [11] and Seeger et al. [12] attributed the Bauschinger effect to long range back stresses generated by the pile-up of dislocations at microstructural barriers such as grain boundaries, sessile dislocations and second-phase particles during forward loading. These stresses aid reverse dislocation motion when the slip direction is changed during reverse loading. In summary both, internal stress and dislocation theories (or intergranular and intragranular effects) are relevant when explaining the Bauschinger effect in polycrystalline materials.

To this end, the magnitude of the Bauschinger effect in single phase alloys is generally dependent on the stacking fault energy such that a greater lowering in yield strength upon reverse loading is associated with lower stacking fault energy values [13]. The significant Bauschinger effect in low stacking fault energy materials is related to the planar nature of their slip and the consequent 
reversibility of their dislocations. Moreover, in Hadfield steel single crystals [14], TWIP steel [2] and brass [15], deformation twinning is usually accompanied by a prominent Bauschinger effect.

Karaman et al. [14] ascribed the twin-related Bauschinger effect to the long range back stresses generated by the dislocation pile-ups at twin boundaries. Gil Sevillano [4] attributed the generation of back stresses to the high strength of the nanometer-sized twin thicknesses. In agreement with [4], a transmission electron microscopy (TEM) study on TWIP steel further clarified that the high strength of the twins is due to the existence of sessile dislocations (the density of which depends on the alloying content) within the twin lamellae [16]. Thus, the above explanation for the observed back stress attributes the enhanced work hardening in TWIP steel to the composite strengthening provided by the "harder" twins and the "softer" austenite matrix.

While the role of the above intragranular sources to the back stress can be analysed by TEM-based local area diffraction experiments, no such work has been undertaken to-date. On the other hand, the evolution of intergranular stresses during the uniaxial loading of Fe-25Mn-3Si-3Al has been characterised by in-situ synchrotron X-ray diffraction [17]. However, the contribution of these intergranular sources to the back stress can only be quantified by in-situ diffraction measurements during cyclic loading (for example - tension-compression, compression-tension or forward-reverse torsion tests).

The internal strain evolution during cyclic loading allows assessment of the contribution of intergranular and intragranular stresses to the Bauschinger effect. The coupling of neutron diffraction (ND) with an in-situ cyclic testing apparatus enables the simultaneous tracking of the changes in the internal strains along with the bulk response. While several in-situ ND experiments during uniaxial cyclic loading were performed on hexagonal close-packed materials (e.g.: [18, 19]), the very limited studies undertaken on single phase fcc materials have only focussed on austenitic stainless steel which deforms solely by slip [20,21]. Consequently, there are no reports of similar experiments on low stacking fault energy fcc materials such as TWIP steel that deforms via concurrent slip and twinning.

The in-situ ND measurements in turn can be further interpreted using an Elasto-Plastic SelfConsistent (EPSC) [22] polycrystal plasticity model. The EPSC model simulates both, the macroscopic stress-strain behaviour as well as the average response of the various grain orientations. While the original EPSC model implements a Voce law and inherently accounts for the intergranular contribution to the back stress, a recent modification incorporating a non-linear kinematic hardening rule applied to the hardening of slip systems captures the effect of intragranular sources on the back stress [21]. In this regard, while the EPSC model has not been applied previously to the reverse loading of TWIP steels, Favier and Barbier [23] have recently attempted to simulate a reverse simple shear deformation via a translated field model. However, the model failed to capture the Bauschinger effect such that the flow stress was overestimated upon load reversal as the model accounted only for intergranular stresses but not for the intragranular stresses. 
Throughout the paper and following the notation used in [21], the term "isotropic hardening" refers to the Voce law imposing a non-directional, monotonically increasing hardening of deformation systems. It is emphasised that unlike the classical definition of isotropic hardening, the use of the term "isotropic" from here on does not refer to a proportional expansion of the single crystal yield surface while retaining its shape. While such proportional expansion of the yield surface requires that the rate of increase in the critical resolved shear stress is the same for all deformation systems, it is not enforced here during EPSC modelling. Rather, a Voce law with different parameters for each deformation mode will be used.

The classical mechanics definition of kinematic hardening is synonymous with a rigid translation (or displacement) of the entire yield surface and does not enable the prediction of general strain path changes. In the modified EPSC scheme, only the active facet and its opposite facet in the single crystal yield surface are displaced in the same direction, while the inactive facets are not displaced. This approach is more representative of the real material behaviour since it correlates with the reversal of dislocations on a given slip plane [20]. Consequently, the non-linear kinematic hardening rule empirically accounts for the aforementioned directional-planar mechanisms.

Lastly, it should be stated that a universal physical description of hardening during cyclic loading (or kinematic hardening in general) should also take into account the influence of deformationinduced dislocation microstructures on the intragranular back stress (for example, the composite scheme suggested in $[24,25]$ when evaluating material response at large strains, or more complex strain path changes, or for numerous cycles. However, the present experiment along with the modified EPSC model strictly deals with uniaxial load reversal at small strains; where the Bauschinger effect is mostly related to the reversal of dislocation motion [21].

With the above outlook in mind, the present study is the first to characterise the Bauschinger effect in TWIP steel using neutron diffraction. It is also the first time that the modified EPSC model is applied on an fcc material that deforms via concurrent slip and twinning. Hence the ND experimental data set further validates the performance of the modified EPSC model. The effect of the initial uniaxial loading direction and the influence of the loading path on the reverse flow stress for the strain levels used in the present study are also discussed.

\section{Experimental procedure}

The nominal composition of the present TWIP steel is $24 \mathrm{Mn}-3 \mathrm{Al}-2 \mathrm{Si}-1 \mathrm{Ni}-0.06 \mathrm{C}$ wt.\%. The cast slab was $52 \%$ hot rolled and then $42 \%$ cold rolled followed by isochronal annealing. The heat treatment included $240 \mathrm{~s}$ of heating to stable temperature followed by $300 \mathrm{~s}$ of soaking time and immediate water quenching. Full recrystallisation was attained after annealing at $850{ }^{\circ} \mathrm{C}$ with a recrystallised grain size of $\sim 7 \mu \mathrm{m}$ [26]. A round tension/compression sample of $7.62 \mathrm{~mm}(0.3 ")$ gage length and 2.54 
$\mathrm{mm}(0.1 ")$ diameter was machined from the fully recrystallised material with its gage length parallel to the rolling direction.

In-situ neutron diffraction measurements were performed at the Spectrometer for Materials Research at Temperature and Stress (SMARTS) diffractometer at the Manuel Lujan Jr. Neutron Scattering Center, LANSCE, Los Alamos National Laboratory [27]. A schematic of the sample and the diffraction geometry is shown in Fig. 1a. The sample is oriented at $45^{\circ}$ relative to the incident beam. The two detector banks on either side of the sample are at $\pm 90^{\circ}$ relative to the incident beam and simultaneously record data with diffraction vectors parallel $\left(Q_{\|}\right)$and perpendicular $\left(Q_{\perp}\right)$ to the applied load. The incident neutron beam was defined by $3 \times 3 \mathrm{~mm}^{2}$ boron nitride apertures and each diffraction pattern took $\sim 3000 \mathrm{~s}$ to record.

Cyclic uniaxial tension-compression loading between strain limits of $\pm 1 \%$ were performed using strain control on a purpose-built horizontal Instron load frame [27]. Five complete tensioncompression cycles were performed followed by a sixth tension half cycle. For the first three complete tension-compression cycles, diffraction patterns were acquired at predetermined strain levels. Thereafter, diffraction patterns were measured at the maximum tension, maximum compression and zero unload points for the fourth complete and sixth half cycle. The fifth cycle was skipped due to beam time constraints. Throughout the experiment, an extensometer that spanned the irradiated region remained attached to the sample in order to negate load frame compliance errors.

Data analysis involved single peak fitting using the General Structure Analysis Software (GSAS) [28]. Single peak fitting enables following the response of individual lattice reflections $\{h k l\}$ to deformation and thus directly provides information on intergranular effects. The changes in the individual peak positions during deformation as returned from single peak fitting were used to calculate the $\{h k l\}$ specific lattice strains $\left(\varepsilon_{h k l}\right)$ using:

$$
\varepsilon_{h k l}=\frac{d_{h k l}-d_{h k l}^{0}}{d_{h k l}^{0}}
$$

where, $d_{h k l}$ is the instantaneous lattice spacing at any strain step and $d_{h k l}^{0}$ is the unstrained interatomic spacing. Throughout the paper the lattice strain is presented in units of micro-strain $(\mu \varepsilon)$ where $1 \mu \varepsilon=1 \times 10^{-6}$.

It is emphasised here that the lattice strains are a measure of the average elastic normal strain in the direction of the scattering vector in the grains whose $\{h k l\}$ lattice plane normal is parallel to the scattering vector. In other words, they do not represent the state within a single grain but are an average over a family of grains which fulfil the Bragg scattering condition for a given reflection.

A typical axial diffraction pattern collected before the start of cycling is given in Fig. 1b with the inverse pole figure (IPF) obtained from Rietveld refinement given in Fig. 1c. 


\section{Elasto-plastic self-consistent modelling}

In the original EPSC model developed by Turner and Tomé [22], a single grain (or crystallographic orientation) is associated with a volume fraction and is represented as an ellipsoidal inclusion embedded in and interacting with an infinite homogeneous effective medium that corresponds to the polycrystalline aggregate. While the elastic response of the individual grains is described by the single crystal elastic constants, the plastic response of the individual grains is described by activating the various deformation systems $(s)$ at predetermined values of the critical resolved shear stress (CRSS, $\left.\tau_{c r}^{s}\right)$.

While the inclusion formalism predicts uniform stress and strain within an ellipsoidal domain, the values are different for each grain. As a consequence, the inclusion formalism can inherently capture the effect of the intergranular stress but not the intragranular stress. Consequently, without the incorporation of an intragranular back stress during reverse loading, the original EPSC model only accounts for the contribution of intergranular stress to the Bauschinger effect [22]. Recently, a nonlinear kinematic hardening law was implemented by Wollmershauser et al. [21] into the EPSC framework in order to account for the intragranular effects upon reverse loading at low strain levels. The modified EPSC model successfully simulated the macroscopic stress-strain response and the evolution of the lattice strain during the cyclic loading (tension-compression between strain levels of $\pm 2 \%$ ) of $317 \mathrm{~L}$ austenitic stainless steel which deforms solely by slip.

The EPSC model formulation has been detailed in previous publications $[21,22,29]$ and only a brief description is given here. In order to initiate activity on a particular deformation system in the original EPSC model, the resolved shear stress $\left(m^{s}: \sigma^{c}\right)$ has to first reach its CRSS. Thereafter, the deformation system's activity is sustained as long as the resolved shear stress rate $\left(m^{s}: \dot{\sigma}^{c}\right)$ meets the CRSS rate ( $\left.\dot{\tau}_{c r}^{s}\right)$ as it hardens upon straining [22]. Here $\left(\dot{\sigma}^{c}\right)$ is the stress rate and $\left(m^{s}\right)$ is Schmid tensor. In the modified EPSC model, the intragranular back stress effect is captured by updating the activation conditions to include a back stress term $\left(\tau_{b s}^{s}\right)$ such that:

$$
\begin{aligned}
& \tau_{\text {for }}^{s}=m^{s}: \sigma^{c}-\tau_{b s}^{s} \\
& \dot{\tau}_{\text {for }}^{s}=m^{s}: \dot{\sigma}^{c}-\dot{\tau}_{b s}^{s}
\end{aligned}
$$

The $\tau_{b s}^{s}$ term accounts for kinematic hardening in individual slip systems as it reduces the resolved applied stress by the directional back stress arising from the pile-up of dislocations at various barriers. On the other hand, $\tau_{\text {for }}^{s}$ (originally denoted as the CRSS, $\tau_{c r}^{s}$ ) is the isotropic hardening term which is associated with the non-directional accumulation of obstacles such as forest dislocations and/or deformation twin boundaries. Thus, with the accumulation of strain, the evolution of the $\tau_{\text {for }}^{s}$ term accounts for the reduction in the mean slip path and the increased resistance to further dislocation 
motion. The hardening of $\tau_{\text {for }}^{s}$ for each deformation mode follows an extended Voce hardening rule of the form [30]:

$$
\tau_{\text {for }}^{s}=\tau_{0}^{s}+\left(\tau_{1, \text { for }}^{s}+\theta_{1, \text { for }}^{s} \Gamma\right)\left[1-\exp \left(-\frac{\theta_{0, \text { for }}^{s} \Gamma}{\tau_{1, \text { for }}^{s}}\right)\right]
$$

An analogous relationship is employed to describe the evolution of the back stress [21]:

$$
\tau_{b s}^{s}=\left[\left(\tau_{1, b s}^{s}+x_{0}^{s}\right)+\theta_{1, b s}^{s}\left(\gamma^{s}-\gamma_{0}^{s}\right)\right]\left[1-\exp \left(-\frac{\theta_{0, b s}^{s}\left(\gamma^{s}-\gamma_{0}^{s}\right)}{\left(\tau_{1, b s}^{s}+x_{0}^{s}\right)}\right)\right]
$$

$\tau_{0}^{s}$ and $\tau_{1}^{s}$ are the initial and back extrapolated critical resolved shear stresses and $\theta_{0}^{s}$ and $\theta_{1}^{s}$ are the initial and final asymptotic hardening rates for the forest hardening (for in Eq. (4)) and back stress ( $b s$ in Eq. (5)) formulations. $\Gamma=\sum_{s} \gamma^{s}$ is the total accumulated shear strain on all deformation systems in a grain. The $\gamma_{0}^{s}$ and $x_{0}^{s}$ terms are the strain and stress "memory" parameters and are both initially set to zero during the first forward half cycle.

In what follows, a slip system that is active during the forward loading is referred to as a forward slip system ( $s$ ). If the load is reversed, the slip direction of the forward slip system is reversed and the slip system is denoted as a reverse slip system $(-s)$.

Upon load reversal, the above "memory" parameters are updated such that: (i) the $\gamma_{0}^{s}$ value of the reverse slip system at the start of the reverse half cycle is equal to the total accumulated shear $\left(\gamma_{\text {tot }}^{s}\right)$ of the forward slip system at the end of the forward half cycle. Thus $\gamma_{0}^{s}$ accounts for the high initial hardening rate of the reverse slip system. (ii) The $x_{0}^{s}$ value of the reverse slip system at the start of the reverse half cycle is equal to the positive resolved back stress $\left(\tau_{b s}^{s}\right)$ of the forward system at the end of the forward half cycle. Hence $x_{0}^{s}$ reduces the CRSS of the reverse slip system by $\tau_{b s}^{s}$. It is emphasised that both of the aforementioned "memory" parameters are computed as a function of the strain path and are not fitting parameters ${ }^{1}$. In the absence of intergranular contributions to the back stress, the combined memory effects extend the elasto-plastic transition upon slip direction reversal and are a manifestation of the dislocation-based mechanisms governing the Bauschinger effect [21].

As suggested by Lorentzen et al. [20], the rate of increase of the resolved back stress of the active slip system is equal to the rate of decrease of the resolved back stress of the reverse slip system such that:

\footnotetext{
${ }^{1}$ It should be noted that: (i) the evolution of the CRSS (Eq. 4) depends on the total grain shear $(\Gamma)$ whereas the evolution of the back stress (Eq. 5) is a function of single slip system shear $\left(\gamma^{s}\right)$. (ii) Eq. 5 does not involve an initial CRSS as it is accounted for in Eq. 4. Please see Ref. [21] for further details.
} 


$$
\dot{\tau}_{b s}^{-s}=-\dot{\tau}_{b s}^{s}\left(\text { if } \dot{\gamma}^{s} \neq 0\right)
$$

The kinematic hardening rules denoted by Eqs. (5 and 6) are strictly valid for slip systems only as dislocations can reverse their slip direction upon load reversal. It follows that Eqs. (5 and 6) are inapplicable to twinning due to its unidirectional nature.

Lastly, the activities on the various slip and twinning systems harden other each other according to:

$$
\dot{\tau}^{s}=\sum_{s^{\prime}} V^{s} h^{s s^{\prime}} \dot{\gamma}^{s^{\prime}}
$$

where, depending on the hardening law $V^{s}=d \tau^{s} / d \Gamma$ or $d \tau^{s} / d \gamma^{s}$ and $h^{s s^{\prime}}$ is the latent hardening matrix. The hardening matrix has diagonal values (or the self-hardening) of one. In the current work, the off-diagonal values were also set to one such that all deformation systems are assumed to contribute equally to the hardening of each other, i.e.- equal latent hardening is assumed ${ }^{2}$.

While in the original EPSC model [22] twinning is treated as a directional slip mechanism, the present study utilises the "twinning scheme" which has been incorporated into the EPSC model by Clausen et al. [29]. This scheme accounts for: (i) the volume effect of twinning on texture evolution and, (ii) the stress relaxation associated with the twin formation. With regard to the volume effect, the reorientation by twinning for the low strain levels employed in this study is rather limited. Consequently, the volume effect of twinning on the texture evolution at these strain levels can be presumed to be insignificant. In fact, the volume effect of twinning in fcc polycrystals is generally limited even at higher strains [31,32]. Alternatively, the stress relaxation effect is achieved via the so called "finite initial fraction" approach; wherein the twin is assumed to grow to a fixed volume fraction of its parent grain at the nucleation stage. In this scheme, the localised shear transformation associated with the twinning system generates a back shear stress on the twin due to the constraint of the surrounding grains. Due to this effect, the shear in the parent grain is somewhat relaxed while the shear in the newly created twin domain is reversed.

In the present simulations, $24\{111\}\langle 110\rangle$ perfect slip systems (counting both forward and reverse slip directions) and $12\{111\}\langle 112\rangle$ forward twinning systems (as twinning is only operative in one direction) were introduced into the EPSC model. Additionally, the kinematic hardening parameters for twinning were set to zero.

Further EPSC model inputs include the initial sample texture and the single crystal elastic constants. The orientation distribution function (ODF) obtained from X-ray diffraction in [26] was discretised into 5000 single orientations with varying volume fractions chosen to reproduce the initial texture. Since the single crystal elastic constants of the present TWIP steel are not known, interpolated values $\left(C_{11}=161.5 \mathrm{GPa}, C_{12}=110.3 \mathrm{GPa}\right.$ and $C_{44}=133.3 \mathrm{GPa}$, leading to an elastic anisotropy factor $(A)$

${ }^{2}$ We have also verified that suppressing latent hardening for coplanar systems $\left(h^{s s^{\prime}}=0\right)$ reduces the flow stress but does not lead to qualitative changes in the predicted stress-strain response during unloading. 
$\left.=2 C_{44} /\left(C_{11}-C_{12}\right)=5.2\right)$ were estimated from the $a b$-initio calculations of the elastic properties of Fe$\mathrm{Mn}-\mathrm{Al} / \mathrm{Si}$ alloys [33].

\section{Experimental results}

\subsection{Macroscopic behaviour during cyclic loading}

The macroscopic stress-strain response obtained during cyclic loading is shown in Fig. 2a whereas the maximum stress $\left(\sigma_{\max }\right)$ at the end of each tension and compression half cycle is extracted from Fig. $2 \mathrm{a}$ and shown in Fig. $2 \mathrm{~b}$ as a function of the number of half cycles. All macroscopic stresses and strains are shown using their true values. The stress relaxation observed in Fig. 2a corresponds to the period of neutron data collection where the sample was hold at constant strain.

From Figs. 2a and b, it is clearly evident that the increase in the macroscopic flow stress upon multiple tension-compression cycling approaches saturation by the sixth cycle. It is also noted that the maximum stress during the compression half cycles is $\sim 7 \%$ higher than the maximum stress during tension half cycles throughout the experiment.

In Fig. 2a, the relatively sharper elasto-plastic transition during macro yielding ( $290 \mathrm{MPa})$ in the first tensile half cycle is markedly different from the more gradual elasto-plastic transitions recorded when multiple cycling between tension-compression or compression-tension is undertaken. More importantly, the maximum stress at the end of the first tension half cycle is $\sim 320 \mathrm{MPa}$. Upon load reversal, yielding occurs during the unloading and before the macroscopic stress crosses the abscissa at $\sim+100 \mathrm{MPa}$. This is also associated with an anelastic strain $\left(\varepsilon_{\text {anelastic }}\right)$ of $\sim 0.04 \%$ during unloading from either tension or compression half cycles (illustrated in Fig. 2a using a dashed line when unloading from tension to compression). All of the above observations are manifestations of a pronounced Bauschinger effect.

A more quantitative estimation of the Bauschinger effect is obtained by the method originally suggested by Cottrell [34] and implemented by Kuhlmann-Wilsdorf and Laird [35]. Here the cyclic flow stress is divided into a friction stress and a back stress. The friction stress is independent of the direction of loading and is described as the stress associated with short-range interactions such as lattice friction and forest hardening effects. On the other hand, the back stress arises from the intragranular and intergranular stress contributions and aids yielding upon reverse loading. In this approach, the hysteresis loop is considered to be symmetrical such that the small differences in the stress levels between forward and reverse loading half cycles are neglected. Friction $\left(\sigma_{\mathrm{F}}\right)$ and back ( $\sigma_{\mathrm{B}}$ ) stresses are determined as follows:

$$
\sigma_{\max }=\sigma_{\mathrm{F}}+\sigma_{\mathrm{B}}
$$




$$
\sigma_{\mathrm{R}}=\sigma_{\mathrm{F}}-\sigma_{\mathrm{B}}
$$

where, $\sigma_{\max }$ is the maximum stress at the forward loading half cycle and $\sigma_{\mathrm{R}}$ is the yield stress after load reversal taken as the point of deviation from elasticity. Consequently, $\sigma_{\mathrm{B}}$ is defined as:

$$
\sigma_{\mathrm{B}}=\frac{\sigma_{\max }-\sigma_{\mathrm{R}}}{2}
$$

For example, applying Eq. (10) after the first tension half cycle with $\sigma_{\max }=320 \mathrm{MPa}$ and $\sigma_{\mathrm{R}}=100$ MPa results in a back stress of $210 \mathrm{MPa}$. This value of back stress is $\sim 65 \%$ of the maximum stress at the first tension half cycle. The evolution of the back stress with further cycling is also shown in Fig. $2 \mathrm{~b}$. The back stress to maximum stress ratio decreases slightly from $\sim 65 \%$ to $\sim 62 \%$ after the sixth cycle.

\subsection{The evolution of lattice strains}

The evolution of the lattice strains during the first tension half cycle is given in Fig. 3a for various grain families. A linear fit was applied to the initial elastic response (up to $\sigma<150 \mathrm{MPa}$ ) of each grain family in both, axial and radial directions in order to obtain the material diffraction elastic constants listed in Table 1. Typical of anisotropic fcc crystals, the $\{111\}$ and the $\{200\}$ grain families bound the response of other grain families as the $\{111\}$ orientations are the stiffest orientations while the $\{200\}$ are the most elastically compliant orientations [36]. Consequently, the $\{111\}$ grain family exhibits the lowest tensile lattice strain in the axial direction during the elastic regime, whereas the $\{200\}$ orientations record the highest tensile lattice strain (Fig. 3a). In other words, the evolution of the lattice strains in the elastic regime is dictated by the elastic anisotropy such that it follows the relative magnitude of the directional elastic modulus [37].

In agreement with the earlier observations on austenitic stainless steel [36], the axial lattice strain response of the $\{200\}$ grain family appears to exhibit the double inflection behaviour (blue curly brackets in Fig. 3a) comprising the three deformation stages of linear elastic loading, gradual transition to plasticity and a second linear stage following complete yielding.

In other general observations related to Fig. 3a, the smaller negative lattice strains that develop in the radial direction (perpendicular to the load axis) are due to Poisson's effects. Additionally, the larger scatter recorded for the $\{220\}$ orientations is associated with their small volume fraction in the initial texture (refer to the IPF in Fig. 1c).

The changes in the lattice strain of the various grain families in the axial direction as a function of the total macroscopic strain for the first three cycles is shown in Fig. 3b. Since the diffraction technique detects only changes in the elastic lattice strain, the measured lattice strain is necessarily proportional to the sum of the type-I and type-II stresses on a particular grain family [19]. As such, the 
representation in Fig. 3b is also an approximation of the load partitioning between the various grain families based on axial direction data. While the $\{111\}$ orientations are the first to cease accommodating elastic strains, the $\{200\}$ orientations exhibit the largest increase in elastic lattice strain through all tension and compression half cycles (cf. Section 6.1).

It is emphasised that the above approximation of the load partitioning between the various grain families does not account for the lattice strains in the radial direction. In this regard, the radial lattice strains are not straightforward to interpret since they comprise grain families with different plane normals parallel to the direction of loading [36].

\subsection{Residual strains}

The residual lattice strains measured at zero macroscopic stress while unloading from each tension and compression half cycle are shown in Figs. 4a and b, respectively. It is recognised that the anelastic effects associated with the early yielding during load reversal and the time relaxation effects during diffraction measurements (cf. Fig. 2a) influence the lattice strain measurements at the zero unload points. However, the demarcation of the residual strains provides an estimate of the intergranular sources of back stress to the overall Bauschinger effect. In the following paragraphs, the positive and negative signs denote tensile $(+)$ and compressive $(-)$ residual strains or stresses.

As seen in Figs. 4a and b, the $\{200\}$ grain family has the highest axial tensile and compressive strains after unloading from the tension and compression half cycles, respectively. Irrespective of the loading direction, these residual strains tend to saturate after the third half cycle (at approximately $+800 \mu \varepsilon$ and $-900 \mu \varepsilon$ ). The axial $\{111\}$ grain family exhibits the smallest residual strain after unloading from both tension and compression half cycles $^{3}$. The $\{220\}$ grain family develops limited compressive strains $(\sim 120 \mu \varepsilon)$ after unloading from the tension half cycles but has higher tensile strains $(\sim 400 \mu \varepsilon)$ upon unloading from the compression half cycles. To serve as an example, the residual tensile (+) strain in the $\{220\}$ family (Fig. $4 \mathrm{~b}$ ) after a compression half cycle will promote early yielding in a subsequent tension half cycle. On the other hand, the residual strains developed in the $\{200\}$ and $\{311\}$ grain families follow the sign of the applied macroscopic stress such that they do not assist in early yielding upon subsequent load reversal.

The recorded residual strains need to be converted to stresses in order to determine the exact contribution of intergranular stress to the observed back stress. If radial data is ignored, an approximate estimation of the magnitude of intergranular stresses can be obtained when the axial residual strain is converted to stress via the measured diffraction elastic constants (Table 1). For

\footnotetext{
${ }^{3}$ After unloading from the fourth compression half cycle, the axial residual strain in Fig. $4 \mathrm{~b}$ for the $\{111\}$ grain family is compressive; which is opposite to the trend seen in the first three cycles. Since additional data points were not acquired beyond the fourth compression cycle, it is not possible to verify whether this change in the sign of the residual strain will persist with further cycling or is associated with data uncertainty.
} 
example, the axial residual strain of the $\{220\}$ grain family after unloading from compression half cycles is $\sim+400 \mu \varepsilon$. This results in an intergranular stress of $\sim+80 \mathrm{MPa}$ that aids yielding upon subsequent tension. Clearly, the effect of this stress on the overall response will be weighted by the volume fraction of the grains contributing to a corresponding peak.

\section{EPSC simulations}

Due to the load relaxation exhibited during the diffraction measurements, the EPSC model was fitted to the stress-strain time averaged values over the period of data collection (shown as solid blue dots in Fig. 5a). The isotropic and kinematic hardening parameters were adjusted until optimal correspondence with the experimental macroscopic stress-strain was achieved. The parameters utilised in the present simulations are listed in Table 2. It should be noted that since the sample had a round cross-section, the position of the rolling normal (ND) and transverse (TD) directions (contained in the section) is unknown. Consequently, while the experimental neutron data comprises one set of radial strains, the EPSC results perpendicular to the load axis are given in the two orthogonal directions that correspond to the cold rolling ND and TD and bound all radial directions. The difference between the ND and TD predictions gives also an indication of the sensitivity of the radial lattice strains to the exact population of the grains probed in the radial direction.

\subsection{The elastic regime}

The EPSC simulated bulk elastic modulus corresponds very well with the measured macroscopic value (Table 1). The predicted axial and radial (given in two orthogonal directions, $E_{N D}$ and $E_{T D}$ ) elastic moduli of the various grain families are also listed in Table 1. The model is generally in good agreement with the experimental diffraction elastic constants. However, discrepancies were found for the $\{111\}$ grain family such that more compliant (lower) and stiffer (higher) values were predicted in the axial and radial directions, respectively. This disparity can be attributed to the imprecise knowledge of the single crystal elastic constants.

For all grain families, the difference between the two orthogonal elastic moduli $E_{N D}$ and $E_{T D}$ is less than 5\%; with the exception of the $\{220\}$ orientations which returned a $13 \%$ difference (cf. Fig. 6 d). In 309 stainless steel, Pang et al. [38] reported a 30\% difference in the slope of the $\{220\}$ orientations between the transverse and the normal directions. Oliver et al. [39] demonstrated that the elastic response of the transverse $\{110\}$ grain family in anisotropic cubic crystals is very susceptible to crystallographic texture as it is controlled by the grain orientations perpendicular to it (i.e.- along the axial loading direction). These grains comprise orientations distributed between $\langle 100\rangle$ and $\langle 110\rangle$ directions. Grains close to $\langle 100\rangle$ are more susceptible to contraction while those close to $\langle 110\rangle$ are 
more prone to expansion. Therefore, the resultant response will be dictated by the exact distribution of the orientations along the load axis which make up the radial $\{110\}$ grain family.

\subsection{The plastic regime}

The EPSC simulated macroscopic stress-strain behaviour is in very good agreement with the bulk experimental data as shown in Fig. 5a. The experimental flow curve is plotted as a dashed line while the solid dots are the time averaged stress and strain values during the collection of the diffraction patterns. The EPSC prediction closely follows the experimental hysteresis loop such that the modelled macroscopic flow stress also tends to saturate with further cycling. The gradual elasto-plastic transition upon load reversal is generally well captured. In agreement with the experimental evidence, the EPSC model predicts the observed tension-compression asymmetry (Fig. 5b) with higher maximum stresses during compression half cycles (see Section 6.4).

The difference between the combined isotropic and kinematic hardening laws and the original single isotropic hardening law ${ }^{4}$ [22] is shown in Fig. 5c. An EPSC simulation performed using only the latter approach predicts a sharper elasto-plastic transition and only a slight Bauschinger effect. The poor agreement of the EPSC simulation with the experimental data upon load reversal is because the original EPSC formulation only captures the effect of the intergranular stress and not the intragranular stress.

The lattice strain predictions from the EPSC model are shown in Figs. 6 and 7. The lattice strains of the various grain families during the first tension half cycle are given in Fig. 6. The model reasonably capture the shape, magnitude and width of the lattice strain hysteresis loops of the various grain families during the first three cycles in both the axial and radial directions (Fig. 7).

Considering that the elastic effects in austenitic steel are dictated by the evolution of the internal strains at low strain levels $(\sim<2 \%)[40]$ and that there is uncertainty in the value of the single crystal elastic constants of the present TWIP steel, the EPSC model still return satisfactory lattice strain predictions for the first tension half cycle (Fig. 6). For example, in spite of the discrepancies between the measured and simulated diffraction elastic constants for the $\{111\}$ orientations (Table 1), better correspondence between the experimental and simulated lattice strains is obtained beyond $150 \mathrm{MPa}$ in both the axial and radial directions (Figs. 6a and b). Here the effect of the above discrepancy is highlighted by the small deviation in the slope of the radial $\{111\}$ hysteresis loop (Fig. 7a).

The evolution of the lattice strain of the $\{311\}$ grain family is very well captured throughout the loading cycles in both the axial and radial directions as seen in Figs. 6a, b, and 7d.

\footnotetext{
4 The Voce parameters (in MPa) used with the single isotropic hardening law (Eq. (4)) are $\tau_{0}=85, \tau_{1, \text { for }}=20, \theta_{0, \text { for }}$ $=1000, \theta_{1, \text { for }}=10$ for slip systems and $\tau_{0}=105, \tau_{1, \text { for }}=20, \theta_{0, \text { for }}=1000, \theta_{1, \text { for }}=10$ for twinning systems.
} 
As a further point of difference between the EPSC simulations and the experimental data, the predictions of the lattice strains for the $\{200\}$ orientations show greater disparities in both the axial and radial directions (Figs. $6 \mathrm{c}$ and d). In the axial direction, the EPSC model predicts three inflections with four stages (indicated by arrows in Fig. 6c) rather than the two inflection response seen in Fig. 3a. The third inflection and the subsequent fourth stage is accompanied by a significant overestimate of the predicted axial strains for the $\{200\}$ grain family. This deviation could be attributed to the greater influence of load relaxation on compliant orientations such as $\{200\}$.

Another disparity in the EPSC model predictions is noted for the $\{220\}$ grain family in the axial direction (Figs. 6c and 7c). Slight under prediction of the axial lattice strains is seen in Fig. 6c along with a leftward shift in the lattice strain hysteresis loops (Fig. 7c). Here the simulated loops are of the correct width but are shifted towards lower lattice strains values such that under and over predictions are obtained for the tension and compression half cycles, respectively. In the EPSC simulated lattice strains, the shift is slightly less during the tension half cycles than the compression half cycles.

\section{Discussion}

\subsection{Diffraction elastic constant, lattice strains and residual lattice strains}

Fe-Mn-Al-Si TWIP steels tend to have lower $C_{11}$ and $C_{12}$ values (cf. Section 3) compared to Fe-Cr-Ni austenitic stainless steel $\left(C_{11}=198-205 \mathrm{GPa}\right.$ and $C_{12}=125-138 \mathrm{GPa}$ ) [41, 42]. This difference is attributed to the so-called magneto-volume effect; i.e.- the coupling of the magnetic energy to the elastic energy of a material [43]. Both $\mathrm{Mn}$ and $\mathrm{Al}$ have a lattice softening effect due to the strong dependence of local magnetic moments on the lattice parameter (or volume) [43]. While such an effect is less pronounced in shear-type elastic constants $\left(C_{44}\right.$ and $\left.1 / 2\left(C_{11}-C_{12}\right)\right)$, it is more prominent in dilation-type elastic constants $\left(C_{11}\right.$ and $\left.C_{12}\right)$ [44]. The lattice softening effect is associated with a significant elastic anisotropy $(A)$ such that the present TWIP steel returned a value of 5.2; which is significantly higher than the average $A$ values of 3.34-3.77 for austenitic stainless steels $[41,42]$. Accordingly, the difference in the measured elastic constants between the stiffest $\{111\}$ and most compliant $\{200\}$ orientations is $\sim 90 \%$ (Table 1) as opposed to the lower values of $60-68 \%$ reported previously for austenitic stainless steels $[36,38]$.

While the elastic anisotropy dictates the evolution of lattice strains in the elastic regime, their evolution in the elastic-plastic transition region is governed by a combination of the elastic and plastic anisotropy of the material. Here grains with a high directional strength-to-stiffness ratio will yield later than grains with a low directional strength-to-stiffness ratio leading to higher lattice strains in the former grains [37]. Defining (i) the directional strength of a particular crystallographic orientations or fibre as the Taylor factor calculated in the fully developed plasticity regime, and (ii) the directional stiffness as the elastic modulus of that fibre in the elastic regime, Wong and Dawson [37] 
demonstrated that for fcc polycrystals with high elastic anisotropy $(A \geq 2)$, the $\{200\}$ and $\{111\}$ grains have the highest and lowest directional strength-to-stiffness ratio, respectively. It follows that the $\{111\}$ grains are the first to yield whereas the $\{200\}$ grains yield last. Consequently, since the $\{111\}$ grain family yield first, it will exhibit an increase in slope because they no longer accumulate elastic strain at the same rate; as some of their total strain is now plastic strain. Alternatively, the $\{200\}$ grains that stay elastic the longest record the largest increase in elastic lattice strain and exhibit a decrease in slope as they have to carry the load shed by the other yielding grains. Thereafter, in the plastic regime the lattice strains of various grain families tend to saturate (with small incremental changes compared to the elastic regime, see also Fig. 3b), such that the relative trends developed during the elastic-plastic transition region persists with further straining.

As shown in Figs. 4a and b, the residual lattice strains in the $\{200\}$ grain family tends to saturate after the third tension/compression half cycle. Pang et al. [38] made a similar observation during the multiple tension loading-unloading of austenitic stainless steel. In that study, relatively large residual axial strains $(\sim+600 \mu \varepsilon)$ were found in the $\{200\}$ grain family after the first tension loading-unloading cycle at $\sim 2 \%$ strain. Thereafter, only small increases were detected upon multiple tension loadingunloading such that a final residual strain of $\sim+800 \mu \varepsilon$ was reached after unloading at $7.2 \%$ strain. The near-plateauing of residual strain values was ascribed to the saturation of elasticity such that the subsequent evolution of lattice strains is controlled by plastic deformation.

\subsection{The modified EPSC model predictions}

Since the modified EPSC model satisfactorily captures the basic experimental features, it emphasises the adequacy of the hardening description via the combined isotropic-kinematic laws. Thus the EPSC model can be used to gain further insight into the underlying deformation mechanisms. The relative slip/twinning activities as a function of total macroscopic strain (up to the maximum load point of the third compression half cycle) are shown in Fig. 8a. Note that the overlap between the tension (T) and compression (C) lines at the top of Fig. 8a is due to the decrease and subsequent increase in total macroscopic strain during unloading and loading. The EPSC model returns higher twinning activity during the tension half cycles and very limited twinning during the compression half cycles. This is ascribed to the directionality of twinning and Schmid factor considerations wherein not all the twinning systems activated during tension are activated during compression [45]. Decreasing twinning activity is also predicted upon multiple cycling. A similar experimental observation was reported in ferritic stainless steel where twinning activity was found to reduce after a few cycles [46]. The model attributes such behaviour to the increasing slip system activity with greater accumulated strain. Since the opposite systems of those newly activated slip systems gets softer, they are easier to activate upon load reversal than activating new twinning systems. Only $\sim 8 \%$ twin volume fraction is predicted at the end of the sixth cycle; which corresponds to $\sim 18 \%$ total macroscopic strain (Fig. 8b). 
This is in agreement with the limited twinning (no value given) observed after cyclic fatigue experiments on TWIP steel [47]. The above limited twin volume fraction is also close to our ViscoPlastic Self-Consistent model prediction of $\sim 6 \%$ (at $18 \%$ tensile strain) following the simulation of the monotonic tensile loading of the same TWIP steel [32].

A final remark on Fig. 8a is that the slip systems remain active during unloading indicating that no real elastic unloading occurs. This sustained deformation system activity can be ascribed to two factors: (i) the incorporation of a back stress in the modified EPSC model which activates slip very early on during unloading and/or, (ii) the stress relaxation effect of the twinning scheme [29]. In order to further examine these factors, a simulation was performed by treating twinning as a directional slip mechanism ${ }^{5}$ such that the stress relaxation effect of the twinning scheme is suppressed. While the simulation results are not shown here, two main differences between the above directional slip approach and the twinning scheme of this study are noted: (i) in the directional slip case, an interval with zero deformation system activity is predicted during unloading and, (ii) the twinning scheme simulation follows the experimental macroscopic stress-strain curve more closely than the directional slip case during unloading. It follows that the sustained deformation system activity is more attributable to the stress relaxation effect of the twinning scheme and the associated back stress that is enforced upon twin creation.

\subsection{The Bauschinger effect}

While the calculation of the $\sigma_{\mathrm{R}}$ value following the Cottrell scheme (Eq. (10)) is based on the deviation from elasticity upon unloading, an alternative approach calculates the $\sigma_{R}$ value at a particular offset reverse strain (for example, at 0.1 or $0.2 \%$ ). Bouaziz et al. [2] used an offset reverse strain of $0.2 \%$ to evaluate the back stress contribution during the reverse shear testing of Fe-22Mn0.6C TWIP steel. Gutierrez-Urrutia et al. [48] applied the same approach to compare their results in an analogous experiment. Although the offset reverse strain method is very sensitive to the value of the chosen reverse strain [49], applying a $0.2 \%$ offset reverse strain to the current results returned a back stress contribution which was $\sim 20 \%$ of the maximum stress at the end of the first tension half cycle. Alternatively, if the results in $[2,48]$ are extrapolated to the strain level employed in the present work, a lower back stress contribution of $\sim 10 \%$ is deduced.

The Bauschinger effect observed in [2] was attributed to the high back stress generated by the dislocation pile-ups at twin boundaries. Accordingly, Gil Sevillano [4] suggested a composite-type deformation pattern such that back stresses proliferate as the thin twin lamellae reinforce the parent austenite matrix. For the first tension half cycle, the low strain limit (1\%) implies limited twinning.

\footnotetext{
5 This approach follows the treatment of twinning in the original EPSC model such that while a twin system requires a CRSS to be activated, it is only operative in one direction.
} 
Hence twinning alone cannot be responsible for the rather pronounced Bauschinger effect observed upon load reversal. Moreover, the magnitude of the intergranular stresses is insufficient to account for the large observed back stress as verified by the failure of the original EPSC model (which only captures intergranular stress) to predict the macroscopic flow stress upon load reversal (Section 4.3 and Fig. 5c). Consequently, other intragranular sources of back stress should be present in the matrix.

The above statement is supported by TEM investigations conducted at the early stages of deformation. During the monotonic tensile loading of Fe-30Mn-3Al-3Si TWIP steel, deformation was dominated by stacking faults and dislocation arrays at $0.19 \%$ strain [50]. Thereafter, limited deformation twinning was observed at $3 \%$ strain along with predominant planar and wavy dislocations. Planar dislocation structures comprising pile-ups and stacking faults are also the characteristic features in 316L austenitic stainless steel at tensile strains $<1.5 \%$ [51]. During the uniaxial cyclic loading (between $\pm 0.4 \%$ strain) of low stacking fault energy Co-base superalloy, TEM observations revealed extensive stacking faults and stacking fault intersections at the end of the third cycle [52]. Rajan and Vandersande [53] pointed out that the stacking fault intersections can act as effective barriers to dislocation motion in low stacking fault energy materials as dislocation pile-ups were detected at these intersections. Accordingly, stacking fault intersections were suggested as a major source of the back stresses encountered in low stacking fault energy single phase alloys [54]. A similar rationale can be applied to the pronounced Bauschinger effect in the current TWIP steel.

An additional source of the back stress associated with low stacking fault energy materials is the energy storage from the stress-induced separation of partial dislocations [13]. It is argued that while partial dislocations are pulled apart during forward loading, they tend to restore their equilibrium separation distance upon unloading. In turn, the stored energy release and the change in slip direction during load reversal can both contribute to the strain relaxation effects (see the anelastic response during unloading in Fig. 2a).

\subsection{Tension-compression asymmetry}

As seen in Figs. 2a, b and reported previously during the cyclic deformation of $\mathrm{Cu}$ [55], $\mathrm{Cu}-\mathrm{Al}$ [56] and $\mathrm{Cu}-\mathrm{Zn}$ alloys [57], asymmetry in the flow stress between tension and compression exists regardless of the initial loading direction. Although a definitive explanation for the existence of tension-compression asymmetry is still lacking in the literature, the phenomenon is expected in materials that deform by twinning due to their inherently lower yield stress in uniaxial tension compared to compression [58]. Using Bishop and Hill- type analysis, Hosford and Allen [58] concluded that the yield stress should be $28 \%$ higher in compression compared to tension for randomly oriented fcc polycrystals. Chin et al. [59] returned a 25\% difference between compression and tension using Taylor- type analysis. 
The EPSC model predicts the asymmetric behaviour between tension and compression very well as it accounts for the directionality of twinning, and Schmid factor considerations. In the present experiment, forward tension followed by reverse compression was applied. However, in order to negate the effect of load reversal, an EPSC simulation was performed with forward compression followed by reverse tension using the initial experimental texture and the Voce parameters listed in Table 2. As seen in Fig. 9a, higher flow stress during compression is predicted irrespective of the forward-reverse loading path. While the first twinning system is activated during forward tension at a tensile stress of $248 \mathrm{MPa}$ at $0.29 \%$ strain, the first twinning activity is detected during forward compression at a compressive stress of $297 \mathrm{MPa}$ at $0.55 \%$ strain. Regardless of the forward-reverse loading path, the relative system activity is similar; with limited twinning activity noted during compression (Fig. 9b).

The higher flow stress observed here during reverse compression (Fig. 2) is different from earlier studies on reverse shear testing wherein the reverse flow stress remained lower than the monotonic shear curve $[2,48]$. It is noted that these reverse shear tests corresponded to relatively high strains and that the observed Bauschinger effect was attributed mainly to twinning. In order to further examine the effect of loading path on the flow stress, an additional EPSC simulation was performed by applying forward-reverse shear strains equivalent to normal strains of $\pm 1 \%$ using the Voce parameters listed in Table 2. The predicted stress-strain curve is shown in Fig. 9c in terms of the equivalent normal stress $\left(\sigma=\sqrt{3} \cdot \tau_{\text {shear }}\right.$, where $\tau_{\text {shear }}$ is the shear stress $)$ and strain $\left(\varepsilon=\gamma_{\text {shear }} / \sqrt{3}\right.$, where $\gamma_{\text {shear }}$ is the shear strain).

Interestingly, and similar to the forward compression-reverse tension scenario (Fig. 9a), the EPSC simulations return a lower flow stress upon reverse shearing (Fig. 9c). Both these results indicate that the observed lower reverse stress is simply a geometrical and loading path effect rather than being a reflection of the microstructural state or the back stress in the matrix. This is also in agreement with El-Danaf et al. [60] who pointed out that unlike the case of uniaxial loading, the twinning systems during simple shear are mainly coplanar with the primary slip systems such that they do not hinder/obstruct dislocation motion as effectively in the latter deformation mode ${ }^{6}$.

\footnotetext{
6 To further verify this assertion, an additional simulation was performed such that the latent hardening coefficients $\left(h^{\text {ss' }}\right.$ ) for coplanar slip and twin systems were set to zero (see Eq. (7)). Trends similar to Fig. 9(c) were obtained with only a slight decrease in the flow stress during both, forward and reverse loading. Expectedly, the latent hardening coefficients have a marginal effect on the stress-strain response for the strain limits employed in this study. 


\section{Conclusions}

In-situ neutron diffraction and EPSC modelling of fully annealed TWIP steel subjected to uniaxial cyclic (tension-compression) loading was undertaken between strain limits of $\pm 1 \%$. The results show that:

(1) The pronounced Bauschinger effect observed upon load reversal (or the early yielding during unloading) is due to a combination of the intergranular residual stresses and the intragranular sources of back stress such as dislocation pile-ups at the intersections of stacking faults. The formation of such stacking faults is promoted by the low stacking fault energy of the present TWIP steel.

(2) The $\{200\}$ grain family has the highest intergranular lattice strains that follow the sign of the loading direction with tensile and compressive strains during the tension and compression half cycles, respectively. These lattice strains tend to level-off after the third loading cycle due to the saturation of elasticity effects. Alternatively, while smaller intergranular lattice strains develop in the $\{220\}$ grain family, their sign is opposite to the loading direction such that the so-generated residual stress will aid yielding upon load reversal.

(3) The present experimental data generally supports the assumption of a combined isotropickinematic hardening description in simulating the macroscopic stress-strain response and the evolution of lattice strains during the cyclic loading of low stacking fault energy materials that deform via concurrent slip and twinning.

(4) The experimentally observed tension-compression asymmetry is satisfactorily captured by the EPSC model as it accounts for the directionality of twinning as well as Schmid factor considerations. It follows that irrespective of the initial loading direction; the simulations expectedly predict lower twinning activity during the compression half cycles.

(5) By changing the loading direction (compression-tension) and the loading path (forward-reverse shear), lower flow stress was predicted upon load reversal. For the strain limits investigated in the current study, both simulations indicate that such lowering in the flow stress is a geometrical or deformation path effect rather than being a reflection of the actual microstructural state.

\section{Acknowledgments}

The authors are grateful to Prof. D. B. Santos of the Federal University of Minas Gerais, Brazil for providing the source material. The access to major research facilities program (AMRFP) is supported by the Commonwealth of Australia under the International Science Linkages program. Dr. Carlos Tomé was fully supported by the U.S. Department of Energy, Office of Basic Energy Sciences, Division of Materials Sciences and Engineering, Project FWP 06SCPE401 under U.S. DOE Contract No. W-7405- 
ENG-36. This work has benefited from the use of the Lujan Neutron Scattering Center at LANSCE, which is funded by the Office of Basic Energy Sciences (DOE), USA and from the partial funding from the Engineering Materials Institute, UOW, Australia. The Los Alamos National Laboratory is operated by Los Alamos National Security LLC under U.S. DOE Contract DE AC52 06NA25396.

\section{References}

[1] Grassel 0, Kruger L, Frommeyer G, Meyer LW. Int. J. Plast. 2000;16:1391.

[2] Bouaziz O, Allain S, Scott C. Scr. Mater. 2008;58:484.

[3] Remy L. Acta Metall. 1978;26:443.

[4] Sevillano JG. Scr. Mater. 2009;60:336.

[5] Allain S, Chateau JP, Bouaziz O. Mat. Sci. Eng. A 2004;387-389 143.

[6] Bouaziz O, Guelton N. Mat. Sci. Eng. A 2001;319-321:246.

[7] Abel A. Mater. Forum 1987;10:11.

[8] Heyn E. Metall. Erz. 1918;22:436.

[9] Polakowski NH, Ripling EJ. Strength and structure of engineering materials. Englewood Cliffs, New Jersey: Prentice-Hall, Inc., 1966.

[10] Hutchings MT, Withers PJ, Holden TM, Lorentzen T. Introduction to characterization of residual stress by neutron diffraction: CRC Press, 2004.

[11] Mott NF. Phil. Mag. 1952;43:1151.

[12] Seeger A, Diehl J, Mader S, Rebstock H. Phil. Mag. 1957;2:323.

[13] Abel A, Muir H. Phil. Mag. 1973;27:585.

[14] Karaman I, Sehitoglu H, Chumlyakov YI, Maier HJ, Kireeva IV. Metall. Mater. Trans. A 2001;32:695.

[15] Stout MG, Rollett AD. Metall. Trans. A 1990;21:3201.

[16] Idrissi H, Renard K, Schryvers D, Jacques PJ. Scr. Mater. 2010 63:961.

[17] Yan K, Carr DG, Callaghan MD, Liss K-D, Li H. Scr. Mater. 2010;62:246.

[18] Cai S, Daymond MR, Holt RA, Oliver EC. Acta Mater. 2011;59:5305.

[19] Wu L, Agnew SR, Brown DW, Stoica GM, Clausen B, Jain A, Fielden DE, Liaw PK. Acta Mater. 2008;56:3699.

[20] Lorentzen T, Daymond MR, Clausen B, Tomé CN. Acta Mater. 2002;50:1627.

[21] Wollmershauser JA, Clausen B, Agnew SR. Int. J. Fatigue 2012;36:181.

[22] Turner PA, Tomé CN. Acta Metall. Mat. 1994;42:4143.

[23] Favier V, Barbier D. Scr. Mater. 2012;66:972.

[24] Mughrabi H. Acta Metall. 1983;31:1367.

[25] Sauzay M. Int. J. Plast. 2008;24:727.

[26] Saleh AA, Pereloma EV, Gazder AA. Mat. Sci. Eng. A 2011;528:4537.

[27] Bourke MAM, Dunand DC, Ustundag E. Appl. Phys. A 2002;74:S1707.

[28] Larson AC, VonDreele RB. General Structure Analysis System (GSAS). Los Alamos National Laboratory Report LAUR 86-748, 2004.

[29] Clausen B, Tomé CN, Brown DW, Agnew SR. Acta Mater. 2008;56:2456.

[30] Tomé CN, Canova GR, Kocks UF, Christodoulou N, Jonas JJ. Acta Metall. 1984;32:1637.

[31] Leffers T, Ray RK. Prog. Mater. Sci. 2009;54 351.

[32] Saleh AA, Pereloma EV, Gazder AA. Acta Mater. 2013;61:2671.

[33] Gebhardt T, Music D, Kossmann D, Ekholm M, Abrikosov IA, Vitos L, Schneider JM. Acta Mater. 2011;59:3145.

[34] Cottrell AH. Dislocations and plastic flow in crystals: Oxford University Press, London, 1953.

[35] Kuhlmann-Wilsdorf D, Laird C. Mat. Sci. Eng. 1979;37:111.

[36] Clausen B. Characterisation of Polycrystal Deformation by Numerical Modelling and Neutron Diffraction Experiments. PhD thesis, Risø National Laboratory, Roskilde, Denmark, 1997.

[37] Wong SL, Dawson PR. Acta Mater. 2010;58:1658.

[38] Pang JWL, Holden TM, Wright JS, Mason TE. Acta Mater. 2000;48:1131.

[39] Oliver EC, Daymond MR, Withers PJ. Acta Mater. 2004;52:1937.

[40] Daymond MR, Tomé CN, Bourke MAM. Acta Mater. 2000;48:553.

[41] Ledbetter HM. Phys. Status Solidi A 1984;85:89.

[42] Mangalick MC, Fiore NF. Trans. Met. Soc. AIME 1968;242:2363. 
[43] Schilfgaarde Mv, Abrikosov IA, Johansson B. Nature 1999;400:46.

[44] Music D, Takahashi T, Vitos L, Asker C, Abrikosov IA, Schneider JM. Appl. Phys. Lett. 2007;91:191904.

[45] Hosford WF. The Mechanics of Crystals and Textured Polycrystals: Oxford University Press, 1993.

[46] Magnin T, Coudreuse L, Fourdeux A. Mat. Sci. Eng. 1984; 63:L5.

[47] Karjalainen LP, Hamada A, Misra RDK, Porter DA. Scr. Mater. 2012;In Press.

[48] Gutierrez-Urrutia I, del-Valle JA, Zaefferer S, Raabe D. J. Mater. Sci. 2010;45:6604.

[49] Moan GD, Embury JD. Acta Metall. 1979;27:903.

[50] Zhang X, Sawaguchi T, Ogawa K, Yin F, Zhao X. 8th European Symposium on Martensitic Transformations, Article Number: 05029. Prague, Czech Republic: EDP Sciences, 2009.

[51] Feaugas X. Acta Mater. 1999;47:3617

[52] Lu Z, Xu Y, Hu Z. Mat. Sci. Eng. A 1999;270:162.

[53] Rajan K, Vandersande JB. J. Mater. Sci. 1982;17:769.

[54] Rajan K. Scr. Metall. 1983;17:101.

[55] Mughrabi H. Mat. Sci. Eng. 1978;33:207.

[56] Abel A, Wilhelm M, Gerold V. Mat. Sci. Eng. 1979;37:187.

[57] Carstensen JV. Structural Evolution and Mechanisms of Fatigue in Polycrystalline Brass. PhD thesis, Risø National Laboratory, Roskilde, Denmark, 1998.

[58] Hosford WF, Allen TJ. Met. Trans. 1973;4:1424.

[59] Chin GY, Mammel WL, Dolan MT. Trans. Met. Soc. AIME 1969;245:383.

[60] El-Danaf E, Kalidindi SR, Doherty R. Int. J. Plast. 2001;17:1245. 